\title{
Hybrid Turbulence Models: Recent Progresses and Further Researches
}

\author{
Hariyo Priambudi Setyo Pratomo ${ }^{1,2, *}$, Fandi Dwiputra Suprianto ${ }^{2}$, and Teng Sutrisno ${ }^{2}$ \\ ${ }^{1}$ Fachgebiet Numerische Berechnungsverfahren im Maschinenbau, Technische Universität Darmstadt, \\ 64293 Darmstadt, Germany \\ ${ }^{2}$ Department of Mechanical Engineering, Petra Christian University, J1. Siwalankerto No.121-131, \\ Surabaya, 60236, Indonesia
}

\begin{abstract}
Turbulence simulation remains one of the active research activities in computational engineering. Along with the increase in computing power and the prime motivation of improving the accuracy of statistical turbulence modeling approaches and reducing the expensive computational cost of both direct numerical and large turbulence scaleresolving simulations, various hybrid turbulence models being capable of capturing unsteadiness in the turbulence are now accessible. Nevertheless this introduces the daunting task to select an appropriate method for different cases as one can not know a priori the inherent nature of the turbulence. It is the aim of this paper to address recent progresses and further researches within a branch of the hybrid RANS-LES models examined by the first author as simple test cases but generating complex turbulent flows are available from experimentation. In particular, failure of a seamless hybrid formulation not explicitly dependent on the grid scale is discussed. From the literature, it is practical that at least one can go on with confidence when choosing a potential hybrid model by intuitively distinguishing between strongly and weakly unstable turbulent flows.
\end{abstract}

Key words: Delay of RANS-to-LES transition, scale resolving scheme, subgrid length scale, turbulent flow, transient simulation.

\section{Introduction}

Although Reynolds-averaged Navier-Stokes (RANS) models continue to be the cornerstone in industrial computations that are often dominated by the calculation of high Reynolds number-turbulent flows, the economical simulation techniques suffer from their downside in sufficiently predicting turbulence unsteadiness. It is well-known that the RANS models dampen unsteadiness or remove instabilities in the flow because of an assumption of too high turbulent eddy viscosity. Thereby, even on a very fine good quality-mesh the RANS models will remain unable to provide the turbulence scales. However when only the information on the mean flow is sufficient for a turbulence simulation, the RANS models are still favoured for their acceptable accuracy.

\footnotetext{
*Corresponding author: pratomo@fnb.tu-darmstadt.de, hariyo_p@petra.ac.id
} 
To alleviate the drawback of the RANS modeling approaches, one can use Direct Numerical Simulation (DNS) or Large Eddy Simulation (LES). Nevertheless, the computational cost of the numerical methods is very high. For the computation of wall bounded flows even for ones of simple geometries using DNS or LES, high resolutionmeshes, i.e. isotropic grids in the massive turbulence regions to directly resolve the strongly anisotropic-largest turbulence structures in the near wall region which are in the order of the boundary layer thickness are a must. Such situations even are aggravated when dealing with high Reynolds number-wall bounded flows where the boundary layer thickness becomes thinner at high Reynolds number, thus making the utilization of DNS and LES persists to be more restricted for the high Reynolds number-flows. That is why the demand for RANS modeling is never any lower in the future even though one is aware of the strong misgivings on the conceptual grounds of RANS [1], and the most valid reason that brings the relentless aspiration to the RANS modeling urgency is that the demise of RANS closures has not happened until today [2]. The readers are referred to Durbin [2] for recent developments of the RANS closures.

As an attempt to unite the supremacies of RANS modeling approach and DNS or LES into a single solution strategy, nowadays we are fortunate with the advent of various hybrid turbulence modeling schemes. Too few to mention are Detached Eddy Simulation (DES) and Very Large Eddy Simulation (VLES). In this paper, recent progresses and potential researches within a class of the hybrid scale resolving schemes are discussed. A failure of a seamless hybrid formulation which is not explicitly dependent on the grid scale is also presented.

\section{Recent progresses and further studies in hybrid turbulence models}

Since the conception of Spalart-Allmaras Detached Eddy Simulation (S-A DES) of Spalart et al. [3] around $20 \mathrm{yr}$ ago, various hybrid RANS-LES models being capable of resolving the turbulence scales and maintaining the more affordable computation at practical Reynolds numbers than DNS and standard LES such as, SST-Delayed Detached Eddy Simulation (DDES) [4], Extra Large Eddy Simulaton (X-LES) [5], Partially Averaged Navier-Stokes (PANS) [6], DDES [7], Embedded Large Eddy Simulation (ELES) [8], Zonal Large Eddy Simulation (ZLES) [9], SST-Scale Adaptive Simulation (SAS) [9], SSTImproved Delayed Detached Eddy Simulation (IDDES) [10], Spalart-Allmaras Zonal Detached Eddy Simulation (S-A ZDES) [11], RANS-Implicit Large Eddy Simulation (ILES) [12], and Stress-Blended Eddy Simulation (SBES) of ANSYS [13], are now available for unsteady simulations. It is important to note here that the names of the models containing the capital letter DES explicitly are DES variants, although this is not necessarily the case as in RANS-ILES, SBES, and X-LES. Furthermore, the capital letters preceding the hybrid model names, e.g. SST-DES, indicate the names of RANS model used as the baseline model in the hybrid formulations. Among the scale resolving schemes, SSTSAS is the only method having an inherently different strategy in the modification of the turbulence transport equation, as compared to the DES forms.

In the above-mentioned hybrid proposals, one can classify the schemes into zonal and non-zonal techniques. All the models (except ELES, S-A ZDES, and ZLES that are flow problem-dependent zonalization) are non-zonal or global approach in the sense that the method itself chooses automatically the simulation mode during the run, and thus predefinitions between RANS and LES regions are avoided prior to the simulation, as explained by Breuer et al. [14]. An opposite way holds for the zonal procedure where the predefinition of the LES and RANS regions prior to the execution of the simulation is decided by the user through the grid design, the determination of an explicit border, or the 
selection of domains not especially related to wall regions [14]. In this regard, the nonzonal technique is more straightforward and practical than the zonal counterpart with respects to reduced efforts from the user side on conceiving different turbulence regions manifested through the grid design. In this paper we focus the progress of the hybrid turbulence models on the group of non-zonal approach.

Within the DES families, a new search for more physically rationalized definitions of the hybrid length scale $\Delta$ in addition to the standard model in DES and DDES, i.e. the maximum of the cell sizes $\Delta_{\max }$, was pioneered by Shur et al. [15] and Deck [16] due to the needs of formulating a more modular DDES for wall bounded- and massively separated turbulent flows and a more agressive DDES being competent to generate fast break-up of modelled turbulence into resolved turbulence length scales. In this respect, a versatile definition of the hybrid length scale $\Delta_{\text {IDDES }}$ for massively separated- and wall boundedturbulent flows and a new formulation of the filter width $\Delta_{\omega}$ of [17] to prevent a delayed development of instabilities in shear layer have been established in IDDES of [15] and S-A ZDES of [16], respectively. Before [15] and [16], the upgrade of DES was dedicated to prevent an improper activation of resolved turbulence scales simulation in the very nearwall region through the development of a new blending or shielding function, $f_{p}$, i.e. to shield the boundary layer region from the attack of grid-induced separation (GIS) when mesh refinement is required, as in Menter and Kuntz [4], and Spalart et al. [7]. Later on, improvements in the hybrid scale resolving schemes were proposed by Gritskevich et al. [10] for IDDES with fine-tuning to the SST background model. The urgency of developing a more adaptable and aggressive hybrid formalism was also addressed by Menter [18]. Following the advances in IDDES, SBES of ANSYS [13], and RANS-ILES of Islam and Thornber [12] were developed recently. In SBES, a new hybrid filter width $\Delta_{\text {SBES }}$ is defined as a maximum numerical function between the cubic root of the cell volume of Deardorff [19] and a corrected maximum of the cell size. A new grid scale is also used in RANSILES but this novel filter width $\Delta_{\text {RANS-ILES }}$ is based on the cell Jacobian as to maintain a smoother, gradual variation over stretched, anisotropic grids [12]. Clearly, these new subgrid length scales $\Delta$ control the level of eddy viscosity and which wavelengths can be directly captured; thus strongly influencing the performance of the advanced turbulence models.

The hybrid turbulence modeling approaches have been widely used in various cases with varying degrees of success. In the literature, depending on the nature of studies chosen either model application or model development, several hybrid scale-resolving schemes exercised on a set of test case to study their performance or single hybrid turbulence model tested on various geometries to analyse the strength of its modelling strategy are not always performed. In many studies where the focus is on product development, it is usually sufficient to only utilize one hybrid scale-resolving scheme where the choice of the hybrid model used can depend on its availability in a flow solver or relevant supporting evidences to the strength of the model given in the literature. To such situations we can cite for example the works of Wang et al. [20] and Xia et al. [21] who studied the performance of a hybrid turbulence model only on a case, i.e. SST-DDES on a control valve [20] and SSTIDDES on a high-speed train [21]. Despite these prevailing stages, there are a number of studies that employed several hybrid scale-resolving schemes on a set of test case during the last five years such as the work of Islam and Thornber [12] that studied the performances of PANS, S-A DDES, SST-DDES, RANS-ILES on circular cylinder, flat plate, NACA4412 airfoil. In the mentioned studies, the capabilities of SST-DDES, SSTIDDES, and the other hybrid models to produce the turbulence scales were well-proven. More importantly, RANS-ILES was found to be the most superior model over PANS, S-A DDES, SST-DDES [12]. Adding more studies which investigated the performances of the hybrid turbulence models, quite recently aggresive performance of SBES has also been 
corroborated by Ravelli and Barigozzi [22], Meraner et al. [23], Straka et al. [24], and Ekman et al. [25] on turbine nozzle guide vane, burner, segmental orifice plate, and truck; respectively. Comparative studies of the performances of SBES and other hybrid model variants, including SST-DDES, SST-SAS, k- $\varepsilon$ DDES on circular cylinder with splitter and hatchback vehicle can be found in Pratomo and Schäfer [26], Buscariolo et al. [27] where SBES was proven to be the most superior over the other hybrid variants. Among the SBES investigations, the study of Straka et al. [24] is the only one that introduced a new blending function, $\mathrm{f}_{\mathrm{p}}$, solely based on the non dimensional wall distance $\mathrm{y}^{+}$instead of the standard blending function exploited by the rest. Even though all the mentioned studies contribute to the valuable knowledge on how the hybrid models perform, they are limited in the sense of the certain cases used. In fact, computational engineers are always faced with various geometries involving the turbulence where the turbulence itself is case-dependent and more importantly the hybrid modelling approaches have differently ingrained strategies for the turbulence treatment. Compelling questions always come up: "how do the hybrid turbulence models perform on various new cases or existing configurations such as circular cylinder with splitter, wall-mounted hemisphere, tandem circular cylinders?" and "which the hybrid models perform better?" Thus, the availability of hybrid scale resolving schemes is not without difficulty. Computational engineers are faced with the formidable task to choose prospective hybrid models which are suited for various cases. Anyway such a situation leads to a positive impact for the computational engineering community. At least in the context of the model application, studies into the performances of the hybrid turbulence models have never ended up. "How do RANS-ILES, SBES perform on a wallmounted hemisphere?" and "how do RANS-ILES, SBES perform on tandem circular cylinders?" for example can be good proposals for future studies as they are relatively young and still not much investigated.

\section{Failure of SST-SAS on a simple configuration}

Reducing the turbulent eddy viscosity is the way to solve the weakness in RANS modeling approaches in order to derive the hybrid or RANS-LES turbulence models. Within the twoequation eddy viscosity RANS models, the formulation of the viscosity contains two turbulence scales, i.e. turbulence kinetic energy $\mathrm{k}$, turbulence frequency $\omega$ or dissipation $\varepsilon$ where the turbulence scales are solved by their transport equations, that is, the k-transport equation, $\omega$-transport equation or $\varepsilon$-transport equation. For the DES variants such as DDES, IDDES, the eddy viscosity reduction is realized in the k-transport equation through a multiplier to the dissipation term of the turbulence kinetic energy. The multiplier in the dissipation term is a length scale limiter or hybrid function which contains the RANS and subgrid-or LES-length scales. The filter width $\Delta$ mentioned many times in this paper resides in the subgrid-length scales where the LES-scale resolutions are strongly determined by the local grid spacings in the filter width definition.

Unlike the DES variants, the strategy of SAS to lower the eddy viscosity is different. In SST-SAS of Menter and Egorov [9], the turbulent eddy viscosity is lessened by revising the $\omega$-transport equation of SST with an introduction of an additional source term $\mathrm{Q}_{\text {SAS }}$ that is a function of flow variables, i.e. shear strain rate tensor $\mathbf{S}$, length scale L, von Karman length scale $\mathrm{L}_{\mathrm{vK}}$, turbulence kinetic energy $\mathrm{k}$, and turbulence frequency $\omega$. The corresponding formulations of the SAS $\omega$-transport equation, the additional source term $\mathrm{Q}_{\mathrm{SAS}}$, and the von Karman length scale $\mathrm{L}_{\mathrm{vK}}$ are given in equations (1) to (3). Interestingly, there is no filter width variable given in the formulation of the additional source term $\mathrm{Q}_{\text {SAS }}$ (see Equation (2)). Accordingly, such a strategy is safer than one of the DES variants from the attack of GIS, an important term (in the scale-resolving scheme simulation) coined by Spalart et al. 
[7], when the mesh refinement is urgent in certain regions. Nevertheless, as the turbulence is case-dependent the functionality of the additional production term $\mathrm{Q}_{\mathrm{SAS}}$ is strongly affected by the flow parameters where the key variables in the QsAs definition are the von Karman length scale $\mathrm{L}_{\mathrm{vK}}$ and the shear strain rate tensor $\mathbf{S}$. For this reason, when the new source term QsAS is zero (owing to the calculation of those two flow solutions) then the $\omega$ transport equation returns back to its original form of SST, meaning that the vortex scales can not be resolved by SST-SAS.

$$
\begin{aligned}
& \frac{\partial \rho \omega}{\partial t}+\frac{\partial}{\partial x_{j}}\left(\rho U_{j} \omega\right)=\alpha \frac{\omega}{k}+P_{k}-\rho \beta \omega^{2}+Q_{S A S}+\frac{\partial}{\partial x_{j}}\left[\left(\mu+\frac{\mu_{t}}{\sigma_{\omega}}\right) \frac{\partial \omega}{\partial x_{j}}\right]+\left(1-F_{1}\right) \frac{2 \rho}{\sigma_{\omega 2}} \frac{1}{\omega} \frac{\partial k}{\partial x_{j}} \frac{\partial \omega}{\partial x_{j}} \\
& Q_{S A S}=\max \left[\rho \zeta_{2} \kappa S^{2}\left(\frac{L}{L_{V \kappa}}\right)^{2}-C \frac{2 \rho k}{\sigma \varphi} \max \left(\frac{1}{\omega^{2}} \frac{\partial \omega}{\partial x_{j}} \frac{\partial \omega}{\partial x_{j}}, \frac{1}{\mathrm{k}^{2}} \frac{\partial k}{\partial x_{j}} \frac{\partial k}{\partial x_{j}}\right), 0\right] \\
& L_{v K}=\frac{\kappa S}{U^{l l}}
\end{aligned}
$$

In the equations, $\sigma_{\omega 2}$ is the $\sigma_{\omega}$ value for the k- $\varepsilon$ regime of the SST model and U' is the second velocity derivative. The readers are referred to the paper of Menter and Egorov [9] for further details.

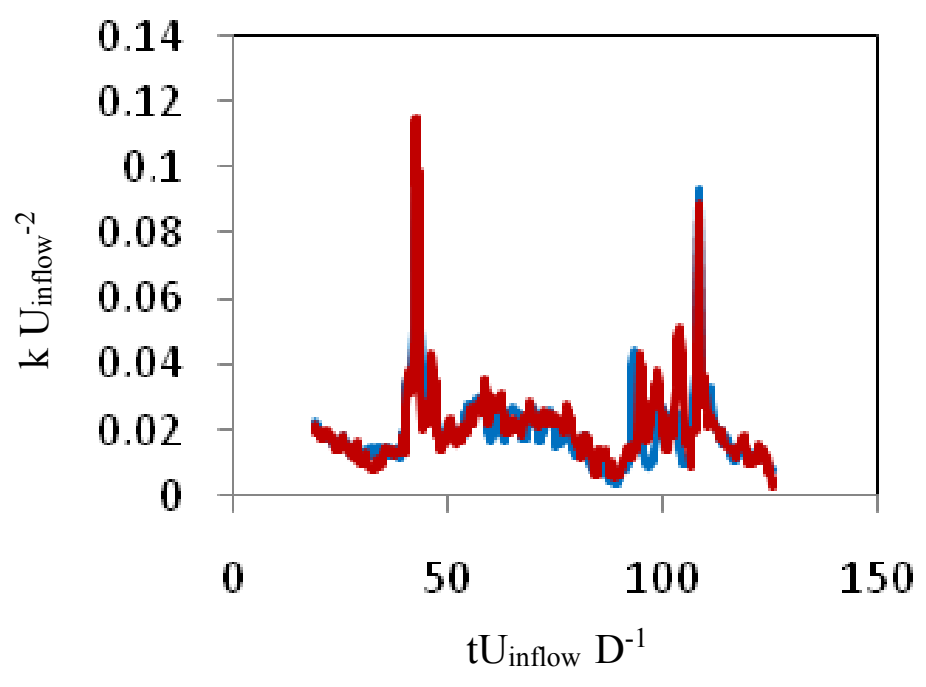

SAS with LES me sh: monitoring point at $z / D=-0.68$

SAS with LES mesh: monitoring point at $z / D=0.68$

(a) 


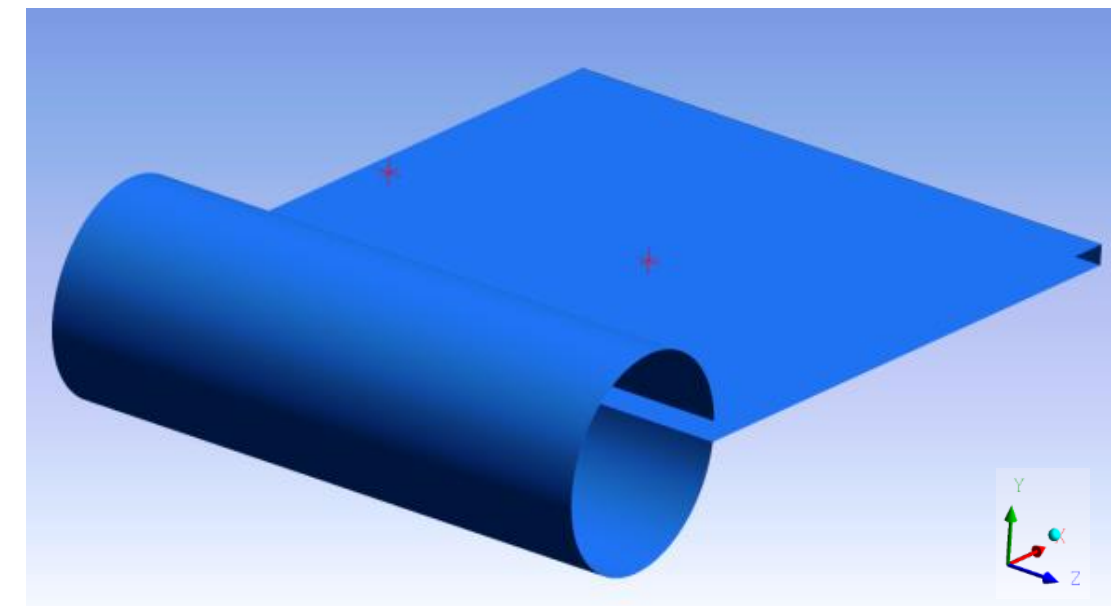

(b)

Fig. 1. (a) Evolution in time of the turbulence kinetic energy k. (b) monitoring points for the turbulence kinetic energy $\mathrm{k}$.

Application of SST-SAS [9] on a benchmark configuration was performed by Pratomo and Schäfer [26]. The configuration considered is a fluid-structure interaction test case of De Nayer et al. [28] where the rubber was treated as a rigid splitter. This was to study the performance of SAS before proceeding with a fluid-structure interaction simulation with a prospective hybrid turbulence model. The Reynolds number of the turbulent flow over the geometry was in a subcritical region in the mode of a transition in the shear layer as in De Nayer et al. [28]. The boundary conditions used inlet, no-slip wall (for the cylinder and the splitter), outlet, slip walls, periodicity for the lateral walls where low turbulence intensity and eddy viscosity ratio were imposed at the inlet. An LES mesh of $14 \times 10^{6}$ control volumes with a growth rate of $1.05, \Delta \mathrm{y}^{+}<5, \Delta \mathrm{x}^{+}=40$, and $\Delta \mathrm{z}^{+}=64$ was crafted for the simulation with a subset case-domain. The subset domain was used as two-point correlations dropped towards zero value within the subset domain [29]. Following Garcia-Villalba et al. [30], two monitoring points for the turbulence kinetic energy $\mathrm{k}$ were added in the wake region behind the circular cylinder (illustrated in Figure $1 \mathrm{~b}$ ) to conclude the evolution in time of the turbulence kinetic energy $\mathrm{k}$ for the requirement of non-dimensional advection time $\mathrm{t}^{*}\left(=t \frac{U_{\mathrm{inflow}}}{D}>100\right)$ and the start of transient statistics averaging period. The progress of the

turbulence kinetic energy $\mathrm{k}$ in time is depicted in Figure 1a for 125 non-dimensional advection times. From the figure the distribution of the turbulence kinetic energy $\mathrm{k}$ in time is already settled, indicating that the turbulent flow was fully developed and ready for the averaging phase.

Figure 2a illustrates the turbulence scales (colored with CFL number) modeled by SSTSAS of Menter and Egorov [9], captured at the non-dimensional advection time of 125. It is demonstrated that SST-SAS [9] failed to sufficiently resolve the vorticity scales on the geometry, even with the LES quality mesh and a very small timestep size $\Delta \mathrm{t}$ of $2.5 \times 10^{-5} \mathrm{~s}$ corresponding to the CFL number of less than 1 [26]. The scale-resolving simulation on the test case just produced a RANS like-solution. Moreover, using different non-dissipative advection schemes and eddy viscosity limiters under the fine temporal and grid resolutions also did not offer any helps, as reported by Pratomo and Schäfer [26]. The fine mesh resolutions simply produced the shear strain rate $\mathbf{S}$ and the von Karman length scale $\mathrm{L}_{\mathrm{vK}}$ that eventually neglected the additional source term $Q_{\text {SAS }}$ in the $\omega$-transport equation of 
SST-SAS, that is, $\mathrm{Q}_{\mathrm{SAS}} \approx 0$ 0. From Equation (2), it is clear that when the argument $\rho \zeta_{2} \kappa S^{2}\left(\frac{L}{L_{V \kappa}}\right)^{2}-C \frac{2 \rho k}{\sigma \varphi} \max \left(\frac{1}{\omega^{2}} \frac{\partial \omega}{\partial x_{j}} \frac{\partial \omega}{\partial x_{j}}, \frac{1}{k^{2}} \frac{\partial k}{\partial x_{j}} \frac{\partial k}{\partial x_{j}}\right)$ is never bigger than 0 , the numerical function "max" in the QSAS definition will take 0 as the largest value thus making $\mathrm{Q}_{\mathrm{SAS}}=0$.

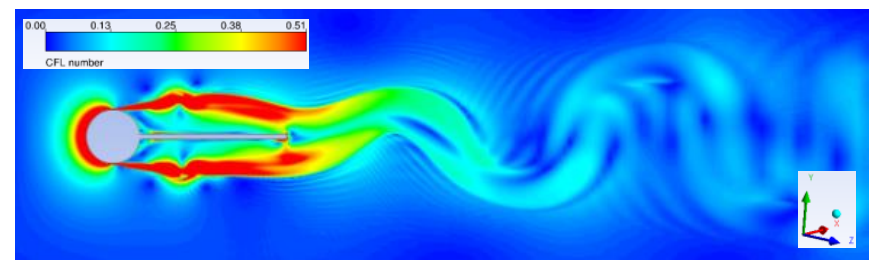

(a)

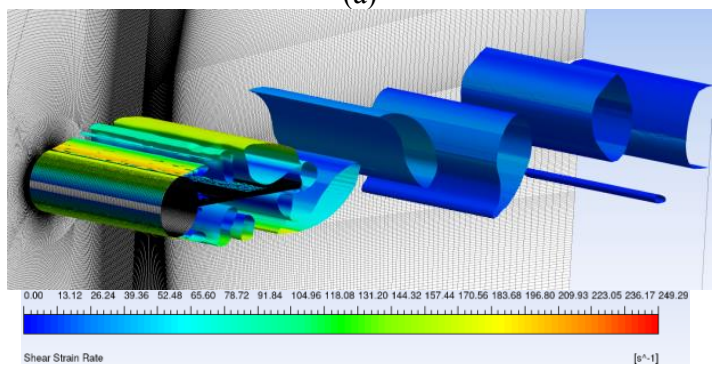

(b)

Fig. 2. (a) Turbulence scales colored with CFL number from the Q-criterion. (b) Contour of the shear strain rate tensor. (Reproduced from Pratomo and Schäfer [26]).

Figure $2 \mathrm{~b}$ displays the contour of the shear strain rate tensor $\mathrm{S}$ which, in this case, contributed to the removal of the additional source term QsAs from Equation (1). It is seen that the existence of the splitter behind the cylinder significantly increased the shear strain rate tensor $\mathbf{S}$ in the wake region (shown in the red color). This is the source of the neglection of the argument ${ }^{2}\left(\frac{L}{L_{k}}\right)^{2}-C^{2 \rho k} \quad\left(\begin{array}{cccccc}1 & \partial \omega & \partial \omega & 1 & \partial k & \partial k\end{array}\right)$ in Equation (2) as $\rho \zeta_{2} \kappa S^{2}\left(\frac{L}{L_{V \kappa}}\right)^{2}-C \frac{2 \rho k}{\sigma \varphi} \max \left(\frac{1}{\omega^{2}} \frac{\partial \omega}{\partial x_{j}} \frac{\partial \omega}{\partial x_{j}}, \frac{1}{k^{2}} \frac{\partial k}{\partial x_{j}} \frac{\partial k}{\partial x_{j}}\right)$
first term gives a small value for the first term as a result of bigger value of the von Karman lengthscale $\mathrm{L}_{\mathrm{vK}}$ (due to the shear strain rate tensor $\mathrm{S}$, see Equation (3)).

\section{Conclusion and outlook}

Recent progresses and further applied researches within the non-zonal hybrid (RANS-LES) turbulence modelling formulation have been addressed in this paper. The non-zonal RANSLES model family is easier to use and more efficient than the zonal hybrid turbulence model group in the sense that RANS and LES modes are automatically activated by the non-zonal technique during the run and much effort to predefine different turbulence regions during the grid design is avoided. To this day, the improvements in the non-zonal RANS-LES proposal embrace several crucial aspects which make the approach remain attractive and advantageous with respect to the computational cost and accuracy. In addition to the extension to various RANS models as the background model for the nonzonal hybrid formulation, the numerical method has been equipped with strong shielding functions and better definitions for the filter width, thus keeping the flexibility and 
aggressive performance of the non-zonal RANS-LES approaches for simulating turbulent flows. From the recent works, two young non-zonal hybrid models appear to be superior among their predecessors. They are SBES and RANS-ILES, as mentioned in this paper. It therefore will be of a strong interest to study the effect and weakness of the differently ingrained strategies in the young hybrid models for the turbulence treatment on various configurations such as circular cylinder with splitter, wall-mounted hemisphere, 3Ddiffuser, tandem circular cylinder as the turbulence is case-dependent. In the long run, this brings a constantly renewed impetus in turbulence research, particularly when the hybrid formulations are married with other disciplines such as acoustics and fluid-structure interaction.

SST-SAS technology is not always more advantageous than RANS models. Proper functionality of the approach directly relies on the inherent nature of turbulence generated by a geometry, not explicitly depends on the grid resolution. This means that a fine spatial resolution carefully crafted for SST-SAS can, otherwise, hinder the production of the turbulence scales by the model because of a certain flow behavior leading to the neglection of the keypart in the additional source term Q QAS in the $\omega$-transport equation of SST-SAS. This is not similar with the DES variants which are explicitly grid dependent-techniques. However, SAS is safer than any DES forms from the GIS phenomena.

Lastly, to reduce volume of the daunting task of deciding prospective hybrid formulations for the turbulence simulation, two important terms have been introduced, i.e. "globally unstable flow" and "locally unstable flow" at least can help the computational engineering community to intuitively choose the potential models suited for certain problems. When a turbulent flow is considered to be globally unstable, the turbulence is strongly unstable. Such a flow type potentially occurs in turbulent flow over any obstacles or bluff bodies. Conversely, turbulent wall bounded-flows with backward-facing steps and turbulent free-shear flows emanating from walls can be categorized as locally unstable flow. As a best practice, those hybrid turbulence models can be recommended to handle each of the flow types.

The first author is indebted to Prof. Dr.rer.nat Michael Schäfer who has introduced and trusted him for doing research in turbulence and fluid-structure interaction at Fachgebiet Numerische Berechnungsverfahren im Maschinenbau (FNB), Technische Universität (TU) Darmstadt and gratefully acknowledges the scholarships of Kementerian Riset, Teknologi, dan Pendidikan Tinggi Republik Indonesia and Universitas Kristen Petra. It is expressly stated that major parts of this manuscript were prepared by the first author during his doctoral study at FNB, TU Darmstadt. Finally, insightful comments from reviewers which have strengthened the manuscript as well as deep discussions with: Mr. Martin Straka of Physikalisch-Technische Bundesanstalt (PTB) Berlin, Dr.-Ing. Wolfgang Bauer of ANSYS Germany and Institut für Land- und Seeverkehr - Fachgebiet Fahrzengantriebe of TU Berlin, and Abe H. Lee, PhD, graduate of the Pennsylvania State University, are also gratefully acknowledged.

\section{References}

1. P. Spalart. Reflections on RANS modelling. In: Progress in Hybrid RANS-LES Modelling, NNFM 111. S.-H. Peng et al. (Eds). Berlin Heidelberg: Springer (2009). p. 7-24. https://link.springer.com/chapter/10.1007/978-3-642-14168-3_2

2. P.A. Durbin, Annu. Rev. Fluid Mech., 50:77-103(2018). https://www.annualreviews.org/doi/abs/10.1146/annurev-fluid-122316-045020

3. P.R. Spalart, W.-H. Jou, M. Strelets, S.R. Allmaras. Comments on the feasibility of LES for wings, and on a hybrid RANS/ LES approach. Proceeding of the 1st AFOSR 
International Conference on DNS/LES. C. Liu, Z. Liu (Eds). Columbus: Greyden Press (1997). p. 137-147. https://www.cobaltcfd.com/pdfs/DES97.pdf

4. F.R. Menter, M. Kuntz. Adaptation of eddy-viscosity turbulence models to unsteady separated flow behind vehicles. Symposium on the aerodynamics of heavy vehicles: trucks, buses, and trains. R. McCallen, F. Browand, J. Ross (Eds). Berlin Heidelberg New York: Springer (2004). p. 339-352.

https://link.springer.com/chapter/10.1007/978-3-540-44419-0_30

5. J.C. Kok, H.S. Dol, B. Oskam, H. van der Ven. Extra large eddy simulation of massively separated flows. 42nd Aerospace Sciences Meeting and Exhibit, (Reno, USA, 2004). AIAA Paper 2004-264. https://arc.aiaa.org/doi/10.2514/6.2004-264

6. S. Girimaji, K. Abdol-Hamid. Partially averaged navier stokes model for turbulence: Implementation and validation. 43rd AIAA Aerospace Sciences Meeting and Exhibit, (Reno, USA, 2005). AIAA Paper 2005-502. https://arc.aiaa.org/doi/10.2514/6.2005$\underline{502}$

7. P.R. Spalart, S. Deck, M.L. Shur, K.D. Squires, M.K. Strelets, A. Travin, Theoret. Comput. Fluid Dyn., 20:181-195(2006).

https://link.springer.com/article/10.1007/s00162-006-0015-0

8. D. Cokljat, D. Caradi, G. Link, R. Lechner, F.R. Menter. Embedded LES methodology for general-purpose CFD solvers. 6th International Symposium on Turbulence and Shear Flow Phenomena, (Seoul, Korea, 2009). https://cfd.spbstu.ru/agarbaruk/doc/2009 Cokljat-et-al._Embedded-LES-Methodologyfor-General-Purpose-CFD-Solvers.pdf

9. F.R. Menter, Y. Egorov. Flow Turbulence Combust., 85,1:113-138(2010). https://link.springer.com/article/10.1007/s10494-010-9264-5

10. M.S. Gritskevich, A.V. Garbaruk, J. Schutze, F.R. Menter, Flow Turbulence Combust., 88,3:431-449(2012). https://link.springer.com/article/10.1007/s10494-011-9378-4

11. S. Deck, Theoret. Comput. Fluid Dyn., 26:523-550(2012). https://link.springer.com/article/10.1007/s00162-011-0240-z

12. A. Islam, B. Thornber, Comput. Fluids, 167:292-312(2018). https://www.sciencedirect.com/science/article/abs/pii/S004579301830135X

13. ANSYS 17.2. ANSYS CFX-Solver Theory Guide. Release 17.2. Pennsylvania: ANSYS, Inc. Canonsburg (2016).

https://support.ansys.com/portal/site/AnsysCustomerPortal/template.fss?file $=\% 2 \mathrm{Fprod}$ docu $\% 2$ F $17.2 \% 2$ FANSYS+CFX-Solver+Theory+Guide.pdf

14. M. Breuer, B. Jaffrézic, K. Arora, Theoret. Comput. Fluid Dyn., 22,3-4:157-187(2008). https://link.springer.com/article/10.1007/s00162-007-0067-9

15. M. L. Shur, P. R. Spalart, M. K. Strelets, A. K. Travin, Int. J. Heat Fluid Flow, 29,6: 1638-1649(2008). https://www.sciencedirect.com/science/article/pii/S0142727X08001203

16. S. Deck. Zonal Detached Eddy Simulation, ZDES, ONERA. In:. Notes on Numerical Fluid Mechanics and Multidisciplinary Design. DESider - A European Effort on Hybrid RANS-LES Modelling, 103. R. Haase, M. Braza, A. Revell (Eds). Berlin Heidelberg: Springer (2009). p. 41-43. https://link.springer.com/book/10.1007/978-3$\underline{540-92773-0}$

17. N. Chauvet, S. Deck, L. Jacquin, AIAA J., 45,10:2458-2473(2007).

https://arc.aiaa.org/doi/10.2514/1.28562 
18. Menter, F. R. Stress-Blended Eddy Simulation: a new paradigm in hybrid RANS-LES modelling. In: Hoarau Y., Peng SH., Schwamborn D., Revell A. (eds) Progress in Hybrid RANS-LES Modelling. HRLM 2016. Notes onNumericalFluidMechanics and Multidisciplinary Design, vol 137. Springer, Cham.6th HLRM Symposium, (France, 2016). https://link.springer.com/chapter/10.1007/978-3-319-70031-1_3

19. J. W. Deardorff, J. Fluid Mech., 41,2:453-480(1970). https://www.cambridge.org/core/journals/journal-of-fluid-mechanics/article/numericalstudy-of-threedimensional-turbulent-channel-flow-at-large-reynoldsnumbers/D84769F4A3443E4C87E8878303890999

20. P. Wang, H. Ma, Y. Liu, J. Fluids Eng., 140,8:1-13(2018). https://fluidsengineering.asmedigitalcollection.asme.org/article.aspx?articleID $=267269$ $\underline{0}$

21. C. Xia, X. Shan, Z. Yang, J. Fluids Eng., 139,5:1-12(2017). http://fluidsengineering.asmedigitalcollection.asme.org/article.aspx?articleID $=2599262$

22. S. Ravelli, G. Barigozi, J. Heat Transfer, 140,9:1-14(2018). http://heattransfer.asmedigitalcollection.asme.org/article.aspx?articleID $=2677510$

23. C. Meraner, T. Li, M. Ditaranto, T. Løvås, Int. J. Hydrogen Energy, 43,14:7155-7168(2018).

https://www.sciencedirect.com/science/article/pii/S0360319918304798

24. M. Straka, A. Fiebach, T. Eichler, C. Koglin, Flow Measurement Instrum., 60:124-133(2018).

https://www.sciencedirect.com/science/article/pii/S0955598617303461

25. P. Ekman, R. Gardhagen, T. Virdung, M. Karlsson, SAE Int. J. Commer. Veh., 9,2:217-223(2016). https://saemobilus.sae.org/content/2016-01-8022/

26. Pratomo H.P.S., Schäfer M. Assessment of hybrid turbulence modeling approaches for fluid-structure interaction. 4th International Conference on Computational Engineering (Darmstadt, Germany, 2017). http://www.graduate-schoolce.de/index.php?eID $=$ tx nawsecured $\& \mathrm{u}=0 \&$ file $=$ fileadmin $/$ template $/$ media/files $/$ icce 2 $\underline{017 / \text { icce } 2017 \text { boa.pdf\&t }=1562020986 \& \text { hash }=706896 \mathrm{~b} 22 \mathrm{cc} 8 \mathrm{eb} 05 \mathrm{c} 7242 \mathrm{a} 837 \mathrm{e} 2 \mathrm{~d} 863 \mathrm{f0}}$ $\underline{32 \mathrm{a} 0 \mathrm{a} 04}$

27. Buscariolo F.F., Magazoni F.C., Maruyama F. K., Alves J.C.L., Marcwolf, Volpe L.J.D. Analysis of turbulence models applied to CFD drag simulations of small hatchback vehicle. 25th SAE Brasil International Congress and Display, (Säo Paulo, Brasil, 2016). SAE Technical Paper Series 2016-36-0201 E. https://saemobilus.sae.org/content/2016-36-0201

28. G. De Nayer, A. Kalmbach, M. Breuer, S. Sicklinger, R. Wüchner, Comput. Fluids, 99:18-43(2014).

https://www.sciencedirect.com/science/article/abs/pii/S0045793014001583

29. De Nayer, G., Kalmbach A., Breuer, M. Fluid-structure interaction in turbulent flow past cylinder/plate configuration I (First swiveling mode). [Online] from http://www.kbwiki.ercoftac.org/w/index.php/Abstr:UFR_2-13 (2016) [Acessed on 2 May 2016]

30. M. Garcia-Villalba, N. Li, W. Rodi, M.A. Leschziner, J. Fluid Mech., 627:55-96(2009). $\quad$ https://www.cambridge.org/core/journals/journal-of-fluidmechanics/article/largeeddy-simulation-of-separated-flow-over-a-threedimensionalaxisymmetric-hill/63B081F00DDBFD1CA8D3291DF6D5BA75 